\title{
Efecto de la sal de estradiol, estatus ovárico y condición corporal sobre el porcentaje de preñez en vacas con cría IATF
}

\author{
Armendano, J.I. ${ }^{3}$; González Chaves, S. ${ }^{2}$; Uslenghi, G. ${ }^{1}$; Cabodevila, J.'; Callejas, S.S. ${ }^{1}$
}

${ }^{1}$ Área de Reproducción, Fac. Cs. Vet. Univ. Nac. Centro Prov. Bs.As., Paraje Arroyo Seco s/n, Tandil (7000), Buenos Aires, Argentina. Tel.: 0249-4439850. E-mail: callejas@vet.unicen.edu.ar. ${ }^{2}$ Actividad privada, Grupo CONPAS, Balcarce, Argentina. ${ }^{3}$ Becario CIC, Buenos Aires, Argentina.

\begin{abstract}
Resumen
Armendano, J.I.; González Chaves, S.; Uslenghi, G.; Cabodevila, J.; Callejas, S.S.: Efecto de la sal de estradiol, estatus ovárico y condición corporal sobre el porcentaje de preñez en vacas con cría IATF. Rev. vet. 26: 2, 108-112, 2015. Se realizó un experimento con el objetivo de evaluar el uso de cipionato de estradiol (CPE) al retiro de los dispositivos intravaginales con progesterona (DISP) sobre el porcentaje de preñez (PP) a la inseminación artificial a tiempo fijo (IATF) en vacas con cría, además se determinó la asociación entre condición corporal (CC) y estatus ovárico (EO) al inicio del protocolo, y su relación con el PP. Se utilizaron 91 vacas con cría Aberdeen Angus (AA) y Polled Hereford (PH) con un posparto de 50-80 días. El día 0 se estimó la CC, se realizó ecografía pre-servicio determinando el EO, el cual se clasificó como: folículos menores a $10 \mathrm{~mm}(\mathrm{~F}<10)$, folículos mayores o iguales a $10 \mathrm{~mm}(\mathrm{~F} \geq 10)$ o cuerpos lúteos (CL) y se colocó un DISP de $0,5 \mathrm{~g}$, más $2 \mathrm{mg}$ de benzoato de estradiol (BE). El día 8 se retiró el DISP, se inyectó $0,5 \mathrm{mg}$ de DL-cloprostenol y los animales se distribuyeron aleatoriamente según el EO al inicio, recibiendo $1 \mathrm{mg}$ de CPE al retiro o $1 \mathrm{mg}$ de BE $24 \mathrm{~h}$ después. En la IATF (53-54 h post-retiro) se utilizó semen de dos toros de probada fertilidad. El diagnóstico ultrasonográfico de gestación se realizó 49 días post-IATF. Se observó una asociación entre CC y EO, siendo diferente la proporción de $\mathrm{F}<10$ y $\mathrm{F} \geq 10$ (CC4: $\mathrm{F}<10=66,7 \%-10 / 15-, \mathrm{F} \geq 10=26,7 \%$-4/15-; CC5-7: $\mathrm{F}<10=24,0 \%-18 / 75-, \mathrm{F} \geq 10=60,0 \%-45 / 75-; \mathrm{p}<0,05$ ), sin observarse diferencias en proporción de CL (CC4=6,7\%-1/15-; CC5-7=16,0\%-12/75-; $>>0,05)$. El PP se afectó por la raza/ toro (AA= 48,6\%-36/74-; $\mathrm{PH}=18,7 \%-3 / 16-; \mathrm{p}<0,05)$ y por el $\mathrm{EO}$, siendo mayor cuando había un $\mathrm{CL}(\mathrm{F}<10=35,7 \%-10 / 28-; \mathrm{F} \geq 10=38,8 \%-19 / 49-; \mathrm{CL}=76,9 \%-10 / 13-; \mathrm{p}<0,05)$. No hubo efecto de la sal de estradiol ( $\mathrm{BE}=45,4 \%-20 / 44-; \mathrm{CPE}=41,3 \%-19 / 46-; \mathrm{p}>0,05)$, de la $\mathrm{CC}$ (CC4= 26,7\%4/15-; CC5-7=46,7\%-35/75-; $>>0,05$ ), ni de la interacción. Se concluye que el uso de CPE al retiro no afecta la fertilidad de vacas con cría IATF, que la CC afecta el EO, y que el EO afecta el PP cuando el rodeo se encuentra en anestro.
\end{abstract}

Palabras clave: vaca, anestro posparto, condición corporal, estatus ovárico, estradiol cipionato.

\begin{abstract}
Armendano, J.I.; González Chaves, S.; Uslenghi, G.; Cabodevila, J.; Callejas, S.S.: Effect of estradiol salt, ovarian status and body condition score on the pregnancy rate in suckled beef cows FTAI. Rev. vet. 26: 2, 108-112, 2015. An experiment was carried out with the aim of evaluate the effect of estradiol cypionate (ECP) given at intravaginal progesterone-releasing device (DISP) removal on the FTAI pregnancy rate (PR) in suckled beef cows, as well as to evaluate the association between body condition score (BCS) and the ovarian status (OS) at the beginning of the synchronization protocol and its relationship with the PR. Ninety-one suckled Aberdeen Angus (AA) and Polled Hereford (PH) cows with a 50 to 80 postpartum interval days were used. On day 0 BCS was estimated and the OS was determined by ultrasonography as corpus luteum (CL), follicle $\geq 10 \mathrm{~mm}(\mathrm{~F} \geq 10)$, or $<10 \mathrm{~mm}$ ( $\mathrm{F}<10)$, and a $0.5 \mathrm{~g}$ DISP was inserted with an injection of $2 \mathrm{mg}$ of estradiol benzoate (EB). On day 8 DISPs were removed, $0.5 \mathrm{mg}$ of DL-cloprostenol were injected and animals were randomly divided in two groups, considering the OS, to receive $1 \mathrm{mg}$ of ECP in this moment or $1 \mathrm{mg}$ of EB $24 \mathrm{~h}$ later. For the FTAI (53-54 h post-removal), semen of two bulls of proven fertility were used. The ultrasonographic pregnancy diagnosis was performed 49 days post-FTAI. An association between BCS and OS was found, being different the proportion of $\mathrm{F}<10$ and $\mathrm{F} \geq 10$ (BCS4: $\mathrm{F}<10=66.7 \%-10 / 15-, \mathrm{F} \geq 10=$ 26.7\%-4/15-; BCS5-7:F $<10=24.0 \%-18 / 75-, \mathrm{F} \geq 10=60.0 \%-45 / 75-; \mathrm{p}<0.05$ ), without differences
\end{abstract}


on $\mathrm{CL}$ proportions (BCS4 $=6.7 \%-1 / 15-; \mathrm{BCS} 5-7=16.0 \%-12 / 75-; \mathrm{p}>0.05$ ). The PR was affected by breed/bull $(\mathrm{AA}=48.6 \%-36 / 74-; \mathrm{PH}=18.7 \%-3 / 16-; \mathrm{p}<0.05)$ and by the $\mathrm{OS}$, being higher when a $C L$ was present $(\mathrm{F}<10=35.7 \%-10 / 28 ; \mathrm{F} \geq 10=38.8 \%-19 / 49-; \mathrm{CL}=76.9 \%-10 / 13-; \mathrm{p}<0.05)$. There was not effect of the estradiol salt used ( $\mathrm{EB}=45.4 \%-20 / 44-; \mathrm{ECP}=41.3 \%-19 / 46-; \mathrm{p}>0.05)$, of the $\mathrm{BCS}(\mathrm{BCS} 4=26.7 \%-4 / 15-; \mathrm{BCS} 5-7=46.7 \%-35 / 75-; \mathrm{p}>0.05)$, or the interaction. In conclusion, the use of ECP at the moment of DISP withdrawal has no influence on the fertility of suckled beef cows at FTAI, the BCS influences the OS, and the OS affects the PR in anestrous animals.

Key words: cow, postpartum anestrous, body condition score, ovarian status, estradiol cypionate.

\section{INTRODUCCIÓN}

La inseminación artificial (IA) ha ido difundiéndose cada vez más en los últimos años en los rodeos de cría de Sudamérica, principalmente de la mano de la implementación de programas de inseminación artificial a tiempo fijo (IATF) ${ }^{5}$, los cuales permiten que un gran número de animales sean inseminados en un tiempo prefijado.

Actualmente, los protocolos más empleados en los programas de IATF combinan el uso de un dispositivo intravaginal impregnado con progesterona (DISP) junto con sales de estradiol y agentes luteolíticos, pudiendo emplearse también hormonas como la gonadotrofina coriónica equina y los análogos sintéticos de la hormona liberadora de gonadotrofinas ${ }^{18}$. Dentro de los protocolos de sincronización que combinan estas hormonas, se difundió mayormente en Argentina aquél que consiste en la colocación de DISP más la administración de $2 \mathrm{mg}$ de benzoato de estradiol (BE), retirándose el DISP 7 u 8 días más tarde, momento en el que se inyecta un agente luteolítico. Veinticuatro horas post-retiro se realiza la administración de $1 \mathrm{mg}$ de BE y se implementa la IATF entre las 48 y $56 \mathrm{~h}$ de retirado el DISP.

Es importante considerar que la cantidad de encierres necesarios para realizar el protocolo descrito anteriormente (4 veces) puede constituir una importante limitante desde el punto de vista operativo y además, si las condiciones de trabajo no son las adecuadas pueden exacerbar los signos de estrés de los animales, comprometiendo los resultados finales ${ }^{15}$. En este sentido se han buscado alternativas que permitan realizar la IATF reduciendo el número de encierres de 4 a 3 , para lo cual en una de las opciones propuestas se reemplaza la segunda aplicación de BE por una de cipionato de estradiol (CPE) inyectado en el momento del retiro del DISP ${ }^{6,23}$.

Otra limitante de gran importancia que debe considerarse y que constituye uno de los principales obstáculos de la eficiencia reproductiva en rodeos de cría, es el anestro posparto ${ }^{25}$, ya que si bien los protocolos mencionados anteriormente poseen la capacidad de inducir la actividad sexual en animales anéstricos ${ }^{2}$, el éxito de los programas de IA estará igualmente muy relacionado con la cantidad de vientres cíclicos al inicio del servicio ${ }^{27}$. En este contexto, dentro de los diferentes factores que afectan la ciclicidad posparto en bovinos para carne ${ }^{25,27,29}$, aquéllos vinculados a la nutrición adquieren un papel preponderante, siendo la mala alimentación y la pobre condición corporal (CC) factores que incrementan los efectos negativos asociados a la presencia del ternero y al amamantamiento, bloqueando la actividad ovárica y extendiendo consecuentemente la duración del período de anestro ${ }^{4}$.

En tal sentido, se ha establecido que la $\mathrm{CC}$ al parto es uno de los factores más importantes que afecta la fertilidad del siguiente servicio, siendo recomendable que las vacas multíparas paran al menos en una $\mathrm{CC} 5{ }^{17}$, y las primíparas al menos en una CC6 ${ }^{10}$, ya que se ha observado que con $\mathrm{CC}$ menores, el anestro posparto comienza a prolongarse (escala 1 a $9 ; 1=$ emaciada y $9=$ obesa). Además es importante considerar la evolución de esa CC en las últimas semanas previas al parto ${ }^{24}$, en la primera parte de la lactancia ${ }^{12}$ y al momento del inicio del nuevo servicio ${ }^{24}$. En referencia a tratamientos de sincronización de la ovulación basados en el uso de progesterona $\left(\mathrm{P}_{4}\right)$, se cita que los vientres deberían presentar al inicio del protocolo al menos una CC4, e idealmente una CC5 para poder obtener buenos porcentajes de preñez a la IATF ${ }^{7}$, siendo importante también que se encuentren en un plano de aumento de peso ${ }^{4}$.

Considerando las problemáticas anteriormente planteadas, los objetivos del presente trabajo fueron evaluar en vacas con cría al pie: a) los resultados a la IATF utilizando CPE al momento del retiro del DISP en lugar de BE 24 horas posteriores, como alternativa para reducir el número de encierres; b) la asociación entre la condición corporal y la actividad ovárica (estatus ovárico: EO) al momento del inicio del protocolo; y c) el impacto de estos últimos dos factores mencionados (EO y CC) sobre el porcentaje de preñez a la IATF.

\section{MATERIAL Y MÉTODOS}

Animales. Se utilizaron 91 vacas con cría al pie de raza Polled Hereford ( $\mathrm{PH} ; \mathrm{n}=16)$ y Aberdeen Angus (AA; $n=75)$, con un rango de posparto entre 50 y 80 días.

Estimación de la condición corporal. Se utilizó la escala de 1 a 9 (CC $1=$ emaciada, CC $9=$ obesa) ${ }^{28}$, considerando la CC4 como condición límite ${ }^{22}$. Un solo animal AA presentó una CC3 y fue excluido del traba- 
jo, al considerarse que su respuesta reproductiva sería diferente y no podría ser evaluada por presentarse solo un individuo en tal condición.

Examen ginecológico previo. El tacto pre-servicio se realizó mediante ultrasonografía (Transductor transrrectal $5 \mathrm{MHz}$; Chison D600VET, China) para determinación del EO, el cual se clasificó como: folículos menores a $10 \mathrm{~mm}(\mathrm{~F}<10)$, folículos mayores o iguales a $10 \mathrm{~mm}(\mathrm{~F} \geq 10)$ o cuerpos lúteos (CL).

Tratamientos hormonales y servicio. El protocolo de sincronización de la ovulación empleado se basó en la colocación al día 0 de un DISP con $0,5 \mathrm{~g}$ de $\mathrm{P}_{4}$ (DIB ${ }^{\circledR} 0,5$, Syntex), junto con la administración intramuscular (i.m.) de $2 \mathrm{mg}$ de BE (benzoato de estradiol Syntex ${ }^{\circledR}$ ). Al día 8 se retiraron los DISP, inyectándose $0,5 \mathrm{mg}$ de DL-cloprostenol por vía i.m. (DL-C; Ciclase $\mathrm{DL}^{\circledR}$, Syntex). A su vez, en base al EO, los animales fueron divididos aleatoriamente en dos grupos homogéneos para recibir $1 \mathrm{mg}$ de $\mathrm{CPE}$ en ese mismo momento (grupo CPE0; n=46; Cipiosyn ${ }^{\circledR}$, Syntex) o $1 \mathrm{mg}$ de BE $24 \mathrm{~h}$ post-retiro (grupo BE24; $n=44$ ). La IATF se realizó entre las 53 y 54 horas posteriores al retiro de los DISP, utilizando semen congelado/descongelado de dos toros de probada fertilidad, uno de raza Aberdeen Angus y otro de raza Polled Hereford, los cuales fueron utilizados para inseminar a vacas de la misma raza.

Diagnóstico de gestación. Se realizó por medio de ultrasonografía, a los 49 días post-IATF empleando un equipo Chison ${ }^{\circledR}$ D600VET (China), con un transductor transrrectal de $5 \mathrm{MHz}$.

Análisis estadístico. La asociación entre la CC y el EO fue evaluada categorizando la $\mathrm{CC}$ en $\mathrm{CC}$ límite (CC4) o CC moderada a óptima (CC5-7) ${ }^{22}$, y las proporciones de los distintos EO fueron analizadas en función de estas categorías mediante test exacto de Fisher, empleando el FREQ procedure del Statistical Analysis System $\left(\mathrm{SAS}^{\circledR}\right)$, utilizándose el análisis de los residuos tipificados corregidos para detectar diferencias significativas en las comparaciones múltiples ${ }^{11}$. El porcentaje de preñez fue evaluado en función de las variables predictoras raza/toro, tratamiento, EO, y CC, mediante la construcción de un modelo de regresión logística binaria empleando el LOGISTIC procedure del SAS ${ }^{\circledR}$, utilizando el método por pasos hacia adelante, con un valor $\mathrm{p}<0,05$ como criterio de inclusión de las variables al modelo. Los efectos raza y toro no pudieron diferenciarse por utilizar un toro para las vacas de raza Polled Hereford y otro para las de raza Aberdeen Angus. Para todos los análisis se fijó un nivel de confianza del $95 \%$ $(\alpha=0,05)$.

\section{RESULTADOS Y DISCUSIÓN}

Los animales presentaron una $\mathrm{CC}$ de 5,02 $\pm 0,62$ (promedio $\pm \mathrm{DE}$ ). De la totalidad de animales, un 16,7\%
(15/90) exhibió una CC4, mientras que el resto registró una $\mathrm{CC}$ comprendida entre 5 y 7 (promedio $\pm \mathrm{DE}=5,2$ $\pm 0,4)$. A su vez, el examen ginecológico previo reveló la presencia de una muy baja proporción de animales con CL $(14,4 \%, 13 / 90)$ y una elevada proporción que presentaba $F \geq 10 \quad(54,4 \% ; 49 / 90)$, lo cual sería indicativo de un rodeo que se encuentra bajo un anestro de tipo superficial ${ }^{16}$. Es importante tener en cuenta que el grado de ciclicidad observado fue menor al que generalmente se reporta para vacas con cría en esa CC y con 50-80 días de posparto ${ }^{26}$, sobre todo si se considera la elevada proporción de animales que presentaban una CC moderada a óptima $(83,3 \%, 75 / 90)$.

Ello podría ser indicativo de que los animales del presente trabajo se encontraban en una $\mathrm{CC}$ menor al momento del parto (momento de mayor correlación entre la CC y la fertilidad posparto) ${ }^{17}$, pudiendo ser la CC tomada al inicio del protocolo el reflejo de una mejoría en el plano nutricional, que favoreció la salida de los animales del anestro profundo a medida que transcurría el posparto, y pese a no alcanzarse un alto grado de ciclicidad se llegó a observar una elevada proporción de animales con folículos grandes.

Se determinó una asociación estadísticamente significativa entre el EO al inicio del protocolo de sincronización de la ovulación y la CC (Tabla 1), mostrando los animales con $\mathrm{CC} 4$ una proporción significativamente mayor de $\mathrm{F}<10(\mathrm{p}<0,05)$, contrario a lo que se apreció en los animales con CC5-7, los cuales presentaban $\mathrm{F} \geq 10$ en mayor medida $(\mathrm{p}<0,05)$. Esto concuerda con resultados obtenidos por otros investigadores ${ }^{19}$, quienes observaron que la $\mathrm{CC}$ modificaba la dinámica folicular afectando el pasaje de folículos medianos a grandes. Por otro lado, la proporción de animales con CL no difirió significativamente según la categoría de $\mathrm{CC}(\mathrm{p}>0,05)$.

Con respecto al porcentaje de preñez a la IATF, se obtuvo como resultado un $43,3 \%$ (39/90), que si bien se ubica por debajo al valor promedio reportado en la bibliografía $(50 \%)^{9}$, es similar al observado por otros autores ${ }^{3}$. Se registró efecto significativo de la raza/toro y del EO $(p<0,05)$, no así de las variables tratamiento y CC, ni de las interacciones entre variables $(\mathrm{p}>0,05)$. En la Tabla 2 se exponen los porcentajes de preñez en función de los efectos evaluados.

Los resultados obtenidos utilizando CPE al retiro de los dispositivos en lugar de BE $24 \mathrm{~h}$ después fueron similares a lo observado en trabajos realizados sobre

Tabla 1. Distribución porcentual de los diferentes estatus ováricos (EO) de acuerdo a la categoría de condición corporal.

\begin{tabular}{lcc}
\hline EO & CC límite (CC 4) & CC moderado a óptimo (CC 5-7) \\
\hline $\mathrm{F}<10$ & $66,7 \%(10 / 15)^{\mathrm{a}}$ & $24,0 \%(18 / 75)^{\mathrm{b}}$ \\
$\mathrm{F} \geq 10$ & $26,7 \%(4 / 15)^{\mathrm{a}}$ & $60,0 \%(45 / 75)^{\mathrm{b}}$ \\
$\mathrm{CL}$ & $6,7 \%(1 / 15)^{\mathrm{a}}$ & $16,0 \%(12 / 75)^{\mathrm{a}}$ \\
\hline
\end{tabular}

${ }^{\mathrm{a}, \mathrm{b}}$ valores con superíndices diferentes en una misma fila difieren en $\mathrm{p}<0,05$. 
Tabla 2. Porcentaje de preñez en función de la raza/toro, tratamiento, condición corporal y estatus ovárico.

\begin{tabular}{|c|c|c|c|c|c|}
\hline \multirow{2}{*}{ item } & & \multicolumn{3}{|c|}{ estatus ovárico } & \multirow{2}{*}{ total } \\
\hline & & $\mathrm{F}<10$ & $\mathrm{~F} \geq 10$ & $\mathrm{CL}$ & \\
\hline \multirow{2}{*}{ raza/toro } & AA & $\begin{array}{l}41,7 \% \\
(10 / 24) \\
\end{array}$ & $\begin{array}{c}41,5 \% \\
(17 / 41) \\
\end{array}$ & $\begin{array}{c}100,0 \% \\
(9 / 9) \\
\end{array}$ & $\begin{array}{l}48,6 \%{ }^{\mathrm{a}} \\
(36 / 74) \\
\end{array}$ \\
\hline & $\mathrm{PH}$ & $\begin{array}{l}0,0 \% \\
(0 / 4) \\
\end{array}$ & $\begin{array}{c}25,0 \% \\
(2 / 8) \\
\end{array}$ & $\begin{array}{c}25,0 \% \\
(1 / 4) \\
\end{array}$ & $\begin{array}{c}18,7 \%^{\mathrm{b}} \\
(3 / 16) \\
\end{array}$ \\
\hline \multirow{2}{*}{ tratamiento } & BE24 & $\begin{array}{l}38,5 \% \\
(5 / 13)\end{array}$ & $\begin{array}{l}41,7 \% \\
(10 / 24)\end{array}$ & $\begin{array}{c}71,4 \% \\
(5 / 7)\end{array}$ & $\begin{array}{l}45,4 \% \\
(20 / 44)\end{array}$ \\
\hline & CPE0 & $\begin{array}{l}33,3 \% \\
(5 / 15) \\
\end{array}$ & $\begin{array}{l}36,0 \% \\
(9 / 25) \\
\end{array}$ & $\begin{array}{c}83,3 \% \\
(5 / 6) \\
\end{array}$ & $\begin{array}{l}41,3 \% \\
(19 / 46) \\
\end{array}$ \\
\hline \multirow{2}{*}{ cond.corp. } & límite & $\begin{array}{l}40,0 \% \\
(4 / 10) \\
\end{array}$ & $\begin{array}{l}0,0 \% \\
(0 / 4)\end{array}$ & $\begin{array}{l}0,0 \% \\
(0 / 1) \\
\end{array}$ & $\begin{array}{l}26,7 \% \\
(4 / 15) \\
\end{array}$ \\
\hline & $\begin{array}{l}\text { moderada } \\
\text { óptima }\end{array}$ & $\begin{array}{l}33,3 \% \\
(6 / 18)\end{array}$ & $\begin{array}{c}42,2 \% \\
(19 / 45)\end{array}$ & $\begin{array}{r}83,3 \% \\
(10 / 12) \\
\end{array}$ & $\begin{array}{l}46,7 \% \\
(35 / 75)\end{array}$ \\
\hline total & & $\begin{array}{l}35,7 \% \%^{\mathrm{c}} \\
(10 / 28)\end{array}$ & $\begin{array}{l}38,8 \%^{\mathrm{c}} \\
(19 / 49)\end{array}$ & $\begin{array}{l}76,9 \% \%^{d} \\
(10 / 13)\end{array}$ & $\begin{array}{l}43,3 \% \\
(39 / 90)\end{array}$ \\
\hline
\end{tabular}

a, b Valores con superíndices diferentes en una misma columna difieren en $\mathrm{p}<0,05$. ${ }^{\mathrm{c}, \mathrm{d}}$ Valores con superíndices diferentes en una misma fila difieren en $\mathrm{p}<0,05$.

vacas de raza británica con cría al pie ${ }^{23}$. Esto indicaría que el empleo de CPE al retiro del DISP en lugar del BE $24 \mathrm{~h}$ posteriores sería una opción viable para reducir el número de encierres sin afectar la fertilidad de esta categoría, siendo importante remarcar que el uso de CPE al retiro aportaría una mayor elasticidad en la hora de realización de la IATF en comparación con el uso de $\mathrm{BE}$ en ese mismo momento (otra alternativa propuesta para reducir el número de encierres), ya que si bien los resultados obtenidos por otros autores al comparar estos dos protocolos de 3 encierres han sido equivalentes ${ }^{8,14}$, se observó una caída significativa de la fertilidad cuando se utiliza BE al retiro y la IATF se realiza después de las $50 \mathrm{~h}^{1}$.

Por el contrario, al utilizar CPE al retiro se han obtenido resultados equivalentes luego de realizarse las IATF en rangos horarios comprendidos entre las 48 y $54 \mathrm{~h}$ post-retiro ${ }^{21}$, lo cual le confiere a este protocolo una mayor ventaja práctica, al permitir reducir el número de encierres y además aportar elasticidad en el tiempo de realización de la IATF.

Con respecto al efecto de la $\mathrm{CC}$ al inicio del protocolo sobre el porcentaje de preñez a la IATF, si bien se observó una diferencia numérica importante, el hecho de que la misma no haya sido significativa podría explicarse teniendo en cuenta que sólo un bajo porcentaje de los animales presentó una CC límite $(\mathrm{n}=15)$ por lo cual se perdería potencia en el test estadístico empleado. También habría que considerar que en el presente trabajo todos los animales evaluados se encontraban al menos en una $\mathrm{CC} 4$, la cual ha sido reportada por otros autores como la condición mínima necesaria para alcanzar resultados aceptables a la IATF ${ }^{4}$.

Por otro lado, el mayor porcentaje de preñez observado en los animales que presentaban un CL reflejaría el concepto propuesto por otros autores ${ }^{27}$ sobre la importancia de tener la mayor proporción posible de animales cíclicos al inicio del servicio, ya que si bien se trataba de un rodeo en un anestro superficial, la capacidad inductora del protocolo empleado no fue suficiente para lograr que la respuesta al tratamiento fuese independiente del EO al inicio.

Con respecto al efecto raza/toro observado, si bien los efectos no pudieron discriminarse individualmente en el análisis, debería considerarse que no se han reportado diferencias de fertilidad entre razas británicas para carne ${ }^{13,20}$, debiendo probablemente atribuirse estas diferencias al semen empleado, ya que si bien ambos eran aptos, no debe descartarse la posibilidad que existiesen uno o varios factores que pudiesen afectar la fertilidad del semen y que no hayan sido detectados por las pruebas realizadas de rutina ${ }^{6}$. Igualmente debe considerarse que la cantidad de animales Polled Hereford en el presente trabajo era baja.

Se concluye que bajo las condiciones del presente experimento y en tratamientos que utilicen dispositivos con $0,5 \mathrm{~g}$ de progesterona en combinación con estrógenos y un agente luteolítico, la administración de CPE al retiro del DISP o de BE $24 \mathrm{~h}$ post-retiro no afecta el porcentaje de preñez en vacas con cría, siendo la administración de CPE al retiro una alternativa viable para reducir el número de encierres de 4 a 3. Contrariamente, bajo las condiciones de anestro del experimento, el EO es influyente sobre el porcentaje de preñez a la IATF, preñándose en mayor medida aquellos animales que presentan un CL. Además, el EO guarda relación con la CC, y esta última, tomada al inicio del tratamiento, no siempre refleja exactamente el nivel de ciclicidad del rodeo, reforzándose la necesidad de conocer la $\mathrm{CC}$ al parto y la evolución de la misma, además de la realización del tacto pre-servicio para constatar el estatus reproductivo del rodeo a los fines de implementar programas de IATF de la forma más eficiente posible, sobre todo cuando no se cuenta con los datos de CC mencionados anteriormente.

Agradecimientos: Al Méd. Vet. Ricardo Chayer por la colaboración prestada.

\section{REFERENCIAS}

1. Ayres H, Martins CM, Ferreira RM, Mello JE, Dominguez JH, Souza AH, Valentin R, Santos IC, Baruselli PS. 2008. Effect of timing of estradiol benzoate administration upon synchronization of ovulation in suckling Nelore cows (Bos indicus) treated with a progesteronereleasing intravaginal device. Anim Reprod Sci 109: 77-87.

2. Bó GA, Baruselli PS, Martínez MF. 2003. Pattern and manipulation of follicular development in Bos indicus cattle. Anim Reprod Sci 78: 307-326.

3. Bó GA, Cutaia L, Baruselli PS. 2004. Programas de inseminación artificial y transferencia de embriones a tiem- 
po fijo. Proceedings $1^{\circ}$ Simpósio Internacional de Reprodução Animal Aplicada, Londrina, Brasil, p. 56-81.

4. Bó GA, Cutaia L, Chesta P, Balla E, Pincinato D, Peres LC, Maraña D, Avilés M, Menchaca G, Baruselli PS. 2005. Implementación de programas de inseminación artificial en rodeos de cría de Argentina. Proceedings VI Simposio Internacional de Reproducción Animal, Córdoba, Argentina, p. 97-128.

5. Bó GA, Baruselli PS, Mapletoft RJ. 2013. Synchronization techniques to increase the utilization of artificial insemination in beef and dairy cattle. Anim Reprod Sci 10 : 137-142.

6. Cesaroni G, Butler HM, Durand MJ. 2007. Evaluación del uso de dos ésteres de estradiol dobre la tasa de fertilidad a la IATF en vacas secas, tratadas con un dispositivo intravaginal con progesterona. Rev Taurus 9: 12-18.

7. Cutaia L, Veneranda G, Tríbulo R, Baruselli PS, Bó GA. 2003. Programas de inseminación artificial a tiempo fijo en rodeos de cría: factores que lo afectan y resultados productivos. Proceedings V Simposio Internacional de Reproducción Animal, Córdoba, Argentina, p. 119-132.

8. Cutaia L, Balla E, Bó GA. 2005. Efecto del momento de la administración de benzoato o cipionato de estradiol para inducir la ovulación en vaquillonas tratadas con DIB e inseminadas a tiempo fijo. Proceedings VI Simposio Internacional de Reproducción Animal, Córdoba, Argentina, p. 394.

9. Cutaia L, Peres LC, Pincinato D, Chesta P. 2007. Programas de sincronización de celos en vaquillonas de carne: puntos críticos a tener en cuenta. Proceedings VII Simposio Internacional de Reproducción Animal, Córdoba, Argentina, p. 83-93.

10. Derouen SM, Franke DE, Morrison DG, Wyatt WE, Coombs DF, White TW, Humes PE, Greene BB. 1994. Prepartum body condition and weight influences on reproductive performance of first-calf beef cows. J Anim Sci 72: 1119-1125.

11. Haberman SJ. 1973. The analysis of residuals in crossclassified tables. Biometrics 29: 205-220.

12. Lalman DL, Keisler DH, Williams JE, Scholljegerdes EJ, Mallet DM. 1997. Influence of postpartum weight and body condition change on duration of anestrus by undernourished suckled beef heifers. J Anim Sci 75: 2003-2008.

13. Marshall DM, Monfore MD, Dinkel CA. 1990. Performance of Hereford and two-breed rotational crosses of Hereford with Angus and Simmental cattle: I. Calf production through weaning. J Anim Sci 68: 4051-4059.

14. Martins CM, Castricini ES, Sá Filho MF, Gimenes LU, Baruselli PS. 2005. Dinâmica folicular de vacas nelore tratadas com cipionato ou benzoato de estradiol em protocolos de inseminação artificial em tempo fixo. Acta Scientiae Veterinariae 33 (Supl. 1): 2895.

15. Moberg JP. 1976. Effects of environment and management stress on reproduction in the dairy cow. J Dairy Sci 59: 1618-1624.

16. Morales JT, Cavestany D. 2012. Anestro posparto en vacas lecheras: tratamientos hormonales. Revisión. Veterinaria (Montevideo) 48: 19-27.
17. Morrison DG, Spitzer JC, Perkins JL. 1999. Influence of prepartum body condition score change on reproduction in multiparous beef cows calving in moderate body condition. J Anim Sci 77: 1048-1054.

18. Peralta Torres JA, Aké JR, Centurión FG, Magaña JG. 2010. Comparison of estradiol cypionate and estradiol benzoate effects on ovaric activity, estrus and ovulation on anestrus Bos indicus cows. J Anim Vet Adv 9: 466-470.

19. Perry RC, Corah LR, Cochran RC, Beal WE, Stevenson JS, Minton JE, Simms DD, Brethour JR. 1991. Influence of dietary energy on follicular development, serum gonadotropins, and first postpartum ovulation in suckled beef cows. J Anim Sci 69: 3762-3773.

20. Pexton JE, Farin PW, Rupp GP, Chenoweth PJ. 1990. Factors affecting mating activity and pregnancy rates with beef bulls mated to estrus synchronized females. Theriogenology 34: 1059-1070.

21. Ramos M, Chesta P, Allende R, Mazzeo R, Monti JI. 2009. Efecto de la cantidad de espermatozoides por dosis de semen y el momento de la inseminación sobre la tasa de preñez en vacas y vaquillonas Braford inseminadas a tiempo fijo. Proceedings VIII Simposio Internacional de Reproducción Animal, Córdoba, Argentina. (En CD: Simposio 2009/Sincronización celos inseminación bovinos/43).

22. Richards MW, Spitzer JC, Warner MB. 1986. Effect of varying levels of postpartum nutrition and body condition at calving on subsequent reproductive performance in beef cattle. J Anim Sci 62: 300-306.

23. Rocha DC, Beskow A, De Azeredo DM, Ponsati DM, Kuhl FN, Mattos R, Gregory RM. 2005. Efecto del uso de distintos ésteres de estradiol como inductores de la ovulación sobre la tasa de preñez en programas de inseminación artificial a tiempo fijo. Proceedings VI Simposio Internacional de Reproducción Animal, Córdoba, Argentina, p. 419.

24. Selk GE, Wettemann RP, Lusby KS, Oltjen JW, Rasby RJ, Garmendia JC. 1988. Relationship among weight change, body condition and reproductive performance of range beef cows. J Anim Sci 66: 3153-3159.

25. Short RE, Bellows RA, Staigmiller RB, Berardinelli JG, Custer EE. 1990. Physiological mechanisms controlling anestrus and infertility in postpartum beef cattle. $J$ Anim Sci 68: 799-816.

26. Stevenson JS, Johnson SK, Milliken GA. 2003. Incidence of postpartum anestrus in suckled beef cattle: treatments to induce estrus, ovulation and conception. The Prof Anim Sci 19: 124-134.

27. Stevenson JS. 2004. Puberty and anestrous: dealing with non-cycling females. Proceedings of Applied Reproductive Strategies in Beef Cattle, Nebraska, EEUU, p. 117-130.

28. Wagner JJ, Lusby KS, Oltjen JW, Rakestraw J, Walters LE. 1988. Carcass composition in mature hereford cows: estimation and effect on daily metabolizable energy requirement during winter. J Anim Sci 66: 603-612.

29. Yavas Y, Walton JS. 2000. Postpartum acyclicity in suckled beef cows: a review. Theriogenology 54: 25-55. 